\title{
Isomorphic Operators and Functional Equations for the Skew-Circulant Algebra
}

\author{
Zhaolin Jiang, ${ }^{1}$ Tingting $\mathrm{Xu},{ }^{1,2}$ and Fuliang $\mathrm{Lu}^{1}$ \\ ${ }^{1}$ Department of Mathematics, Linyi University, Linyi, Shandong 276000, China \\ ${ }^{2}$ Department of Mathematics, Shandong Normal University, Jinan, Shandong 250014, China \\ Correspondence should be addressed to Tingting Xu; xutingting655@163.com
}

Received 28 March 2014; Accepted 27 April 2014; Published 8 May 2014

Academic Editor: Tongxing Li

Copyright (c) 2014 Zhaolin Jiang et al. This is an open access article distributed under the Creative Commons Attribution License, which permits unrestricted use, distribution, and reproduction in any medium, provided the original work is properly cited.

\begin{abstract}
The skew-circulant matrix has been used in solving ordinary differential equations. We prove that the set of skew-circulants with complex entries has an idempotent basis. On that basis, a skew-cyclic group of automorphisms and functional equations on the skew-circulant algebra is introduced. And different operators on linear vector space that are isomorphic to the algebra of $n \times n$ complex skew-circulant matrices are displayed in this paper.
\end{abstract}

\section{Introduction}

Skew circulant and circulant matrices have became important tools in solving various differential equations. Bertaccini and $\mathrm{Ng}$ [1] proposed a nonsingular skew-circulant preconditioner for systems of LMF-based ODE codes. Delgado et al. [2] developed some techniques to obtain global hyperbolicity for a certain class of endomorphisms of $\left(R^{p}\right)^{n}$ with $p, n \geq 2$; this kind of endomorphisms is obtained from vectorial difference equations where the mapping defining these equations satisfies a circulant matrix condition. Wilde [3] developed a theory for the solution of ordinary and partial differential equations whose structure involves the algebra of circulants. He showed how the algebra of $2 \times 2$ circulants relates to the study of the harmonic oscillator, the Cauchy-Riemann equations, Laplace's equation, the Lorentz transformation, and the wave equation. And he used $n \times n$ circulants to suggest natural generalizations of these equations to higher dimensions. Using circulant matrix, Karasözen and Şimşek [4] considered periodic boundary conditions such that no additional boundary terms will appear after semidiscretization. In [5], the resulting dense linear system exhibits so much structure that it can be solved very efficiently by a circulant preconditioned conjugate gradient method. Brockett and Willems [6] showed how the important problems of linear system theory can be solved concisely for a particular class of linear systems, namely, block circulant systems, by exploiting the algebraic structure. Circulant matrices were also used to solve linear systems from differential-algebraic equations and delay differential equations; see $[7,8]$.

Skew circulant matrices have important applications in various disciplines including image processing, communications, signal processing, encoding, solving Toeplitz matrix problems, preconditioner, and solving least squares problems. They have been put on firm basis with the work of Davis [9] and Jiang and Zhou [10]. Hermitian and skew-Hermitian Toeplitz systems are considered in [11-13]. Lyness and Sørevik [14] employed a skew circulant matrix to construct $s$ dimensional lattice rules. Spectral decompositions of skew circulant and skew left circulant matrices were discussed in [15]. Compared with cyclic convolution algorithm, the skew cyclic convolution algorithm [16] is able to perform filtering procedure in approximately half of computational cost for real signals. In [17] two new normal-form realizations are presented by utilizing circulant and skew circulant matrices as their state transition matrices. The well-known secondorder coupled form is a special case of the skew circulant form. Li et al. [18] gave the style spectral decomposition of skew circulant matrix firstly and then dealt with the optimal backward perturbation analysis for the linear system with skew circulant coefficient matrix. In [19], a new fast 
algorithm for optimal design of block digital filters (BDFs) is proposed based on skew circulant matrix. Gao et al. [20] gave explicit determinants and inverses of skew circulant and skew left circulant matrices with Fibonacci and Lucas numbers.

Besides, there are several papers on the circulant operator and circulant algebra. Wilde [21] discussed aspects of functional equations obtaining generalizations of odd and even functions in terms of $n$th roots of unity in complex number field. Wilde $[22,23]$ generalized properties of $2 \times 2$ circulant matrices and 2-dimensional complex analysis to $n \times n$ circulant matrices. Wilde [24] displayed algebras of operators which are isomorphic to the algebra of $n \times n$ complex circulant matrices. Chan et al. [25] gave different formulations of the operator, discussed its algebraic and geometric properties, and computed its operator norms in different Banach algebras of matrices. Using these results, they also gave an efficient algorithm for finding the superoptimal circulant preconditioner. Chillag [26] proved that eigenvalues of some generalized circulant matrices and the Matteson-Solomon coefficients of a codeword of a cyclic code are all examples of eigenvalues of elements of semisimple finite-dimensional, commutative algebras. The purpose of Chillag's paper is to exhibit elementary properties of such algebras and to apply these properties in various situations. Brink and Pretorius [27] embed some results on Boolean circulants into the context of relation algebras and then generalised them. Interestingly enough, the route lies in group theory. Chan et al. [28] studied the solutions of finite-section Wiener-Hopf equations, by the preconditioned conjugate gradient method, and gave an easy and general scheme of constructing good circulant integral operators as preconditioners for such equations. Benedetto and Capizzano [29] investigated algebraic and geometric properties of the optimal approximation operator, generalizing those proved in [25] for the basic circulant case. Benedetto and Capizzano [30] considered the superoptimal Frobenius operators in several matrix vector spaces and in particular in the circulant algebra, by emphasizing both the algebraic and geometric properties. Hwang et al. [31] are concerned with the hyponormality of Toeplitz operators with matrix-valued circulant symbols. They established a necessary and sufficient condition for Toeplitz operators with matrix-valued partially circulant symbols to be hyponormal and provided a rank formula for the self-commutator. Several norm equalities and inequalities for operator matrices are proved in [32]. These results, which depend on the structure of circulant and skew circulant operator matrices, include pinching type inequalities for weakly unitarily invariant norms.

In passing, skew-circulant operator and algebra were only used in [32]. And solving differential equations by skew circulant matrices has not been fully exploited (as far as we known, only in [1]). It is hoped that this paper will help in changing this. More work continuing the present paper is forthcoming.

In Section 2, we will prove that a complex $n \times n$ skewcirculant matrix is a matrix representation of the group ring (over $\mathbf{C}$ ) of the skew cyclic group. We also prove that the set of skew-circulants with complex entries has an idempotent basis.

In Section 3, functional equations, whose solutions are functions $\mathbf{C}^{n} \rightarrow \mathbf{C}$, are solved using skew-cyclic and idempotent linear operators on the space (labeled $U$ ) of functions $\mathbf{C}^{n} \rightarrow \mathbf{C}$. Further, we will show that this algebra of linear operators is isomorphic to $n \times n$ skew-circulants.

In Section 4, we display skew cyclic and idempotent linear operators on the space $V$ of functions on $n \times n$ complex skewcirculants. Furthermore, we get a relationship between the operators on $V$ and those on $U$.

In Section 5, we show a linear involution on $V$ whose group ring is isomorphic to $2 \times 2$ complex skew-circulant matrices.

\section{Properties of Skew-Circulant}

An $n \times n$ skew-circulant matrix is a square matrix like the following:

$$
S=\left(\begin{array}{cccc}
x_{0} & x_{1} & \ldots & x_{n-1} \\
-x_{n-1} & x_{0} & \ldots & x_{n-2} \\
-x_{n-2} & -x_{n-1} & \cdots & x_{n-3} \\
\vdots & \vdots & \vdots & \vdots \\
-x_{1} & -x_{2} & \cdots & x_{0}
\end{array}\right)_{n \times n}
$$

Let $S_{n}$ denote the set of skew-circulant matrices with complex entries. Let $\pi$ denote the skew-circulant matrix with $x_{1}=1$ and $x_{j}=0$ for $j \neq 1$. Then $\pi^{l}$ (the $l$ th power of $\pi$, $1 \leq l<n)$ is the skew-circulant matrix with $x_{l}=1$ and $x_{j}=0$ for all $j \neq l, \pi^{0}=I$ ( $I$ is the identity matrix), $\pi^{1}=\pi$, and $\pi^{n}=-I$. So $S$ can be written as

$$
S=\sum_{l=0}^{n-1} x_{l} \pi^{l}
$$

for $x_{0}, x_{1}, \ldots, x_{n-1} \in \mathrm{C}$. In other words, $\pi_{0}, \pi_{1}, \pi_{2}, \ldots, \pi_{n-1}$ form a basis for the set of skew-circulant matrices. Let $\omega$ denote any of the $n$th roots of unit, or $\omega=e^{2 \pi i / n}, \eta=e^{\pi i / n}$, and

$$
y_{l}=\sum_{j=0}^{n-1} \eta^{j} \omega^{l j} x_{j}, \quad \text { for } l=0,1, \ldots, n-1 .
$$

Then, through the eigenvalues of the matrix $\pi_{i}, i=$ $0,1, \ldots, n-1$, we know that the numbers $y_{0}, y_{1}, \ldots, y_{n-1}$ are the eigenvalues of the skew-circulant matrix $S$.

Proposition 1. If $S_{n}$, the set of skew-circulant matrices, has a basis $\pi_{0}, \pi_{1}, \pi_{2}, \ldots, \pi_{n-1}$, then the $S_{n}$ also has another basis $G_{0}, G_{1}, \ldots, G_{n-1}$, where

$$
G_{l}=\frac{1}{n} \sum_{j=0}^{n-1}\left(\eta^{-j} \omega^{-l j} \pi^{j}\right),
$$

for $\omega=e^{2 \pi i / n}, \eta=e^{\pi i / n}$, and $l=0,1, \ldots, n-1$. 
Proof. By calculation, these matrices $G_{l}(l=0,1,2, \ldots, n-1)$ have the following properties:

$$
\begin{gathered}
G_{l}^{2}=G_{l}, \quad \text { for } l=0,1,2, \ldots, n-1 ; \\
G_{l} G_{i}=0, \quad \text { for } l \neq i \\
G_{0}+G_{1}+G_{2}+\cdots+G_{n-1}=I \\
\pi^{l}=\sum_{j=0}^{n-1} \eta^{j} \omega^{l j} G_{j}
\end{gathered}
$$

for $l=0,1, \ldots, n-1$. Thus, the idempotents $G_{0}, G_{1}, \ldots, G_{n-1}$ form a basis for $S_{n}$, and in (2) we also have

$$
S=\sum_{l=0}^{n-1} y_{l} G_{l}=y_{0} G_{0}+\cdots+y_{n-1} G_{n-1} .
$$

We have seen that every skew-circulant matrix, $S \in S_{n}$, can be written in one and only one way in the form (2); that is

$$
S=x_{0} I+x_{1} \pi+x_{2} \pi^{2}+\cdots+x_{n-1} \pi^{n-1}
$$

Proposition 2. If the function $\phi: S_{n} \rightarrow S_{n}$ is defined by

$$
\phi(S)=\eta x_{0} I+\eta \omega x_{1} \pi+\cdots+\eta \omega^{n-1} x_{n-1} \pi^{n-1},
$$

then $\phi^{n}(S)=-S$.

Proof. By composition, we gain that

$$
\begin{aligned}
\phi^{k}(S)= & \eta^{k} x_{0} I+\eta^{k} \omega^{k} x_{1} \pi+\eta^{k} \omega^{2 k} x_{2} \pi^{2} \\
& +\cdots+\eta^{k} \omega^{(n-1) k} x_{n-1} \pi^{n-1}
\end{aligned}
$$

that is, $\phi^{k}$ replaces $x_{l}$ by $\eta^{k} \omega^{l k} x_{l}$ for $l=0,1,2, \ldots, n-1$. Also, $\phi^{n}(S)=-S$.

The function $\phi$ is an automorphism in $S_{n}$ that preserves $\mathbf{C}$, with $\mathbf{C}$ being embedded in $S_{n}$ by the correspondence $c \rightarrow c I$ for $c \in \mathbf{C}$.

Proposition 3. Let $p_{l}$ be the function $S_{n} \rightarrow S_{n}$ defined by

$$
p_{l}=\frac{1}{n} \sum_{j=0}^{n-1} \eta^{-j} \omega^{-l j} \phi^{j}, \quad \text { for } l=0,1, \ldots, n-1
$$

Then,

$$
\begin{gathered}
p_{l}^{2}=p_{l}, \quad \text { for } l=0,1,2, \ldots, n-1 ; \\
p_{l} p_{j}=0, \quad \text { for } l \neq j ;
\end{gathered}
$$

$$
\begin{gathered}
p_{0}+p_{1}+p_{2}+\cdots+p_{n-1}=\phi^{0} ; \\
\eta^{l} p_{0}+\eta^{l} \omega^{l} p_{1}+\cdots+\eta^{l} \omega^{(n-1) l} p_{n-1}=\phi^{l} ; \\
p_{l}\left(x_{0} I+x_{1} \pi+\cdots+x_{n-1} \pi^{n-1}\right)=x_{l} \pi^{l},
\end{gathered}
$$

for $l=0,1, \ldots, n-1$.

Proof. Since $p_{l}=(1 / n) \sum_{j=0}^{n-1} \eta^{-j} \omega^{-l j} \phi^{j}$, we gain that

$$
\begin{aligned}
p_{l}^{2}=\frac{1}{n^{2}}\left[\phi^{0}+\eta^{-1} \omega^{-l} \phi+\cdots+\eta^{-(n-1)} \omega^{-(n-1) l} \phi^{n-1}\right]^{2} \\
=\frac{1}{n^{2}}\left[\phi^{0}+\eta^{-1} \omega^{-l} \phi+\cdots+\eta^{-(n-1)} \omega^{-(n-1) l} \phi^{n-1}\right. \\
+\eta^{-1} \omega^{-l} \phi+\cdots+\eta^{-n} \omega^{-n l} \phi^{n} \\
+\cdots+\eta^{-(n-1)} \omega^{-(n-1) l} \phi^{n-1} \\
\left.\quad+\cdots+\eta^{-2(n-1)} \omega^{-2(n-1) l} \phi^{2(n-1)}\right] \\
=p_{l} .
\end{aligned}
$$

Similarly, we can obtain $p_{l} p_{j}=0$ for $l \neq j$. And, by calculation, we have

$$
\begin{aligned}
p_{0}+ & p_{1}+\cdots+p_{n-1} \\
= & \frac{1}{n}\left[\sum_{j=0}^{n-1} \eta^{-j} \phi^{j}+\cdots+\sum_{j=0}^{n-1} \omega^{-(n-1) j} \eta^{-j} \phi^{j}\right] \\
= & \frac{1}{n}\left[\sum_{j=0}^{n-1} \eta^{-j} \phi^{j}\left(\omega^{0}+\omega^{-j}+\cdots+\omega^{-(n-1) j}\right)\right] \\
= & \frac{1}{n}\left[\sum_{j=0}^{n-1} \eta^{-j} \phi^{j}\left(\frac{1-\omega^{-n j}}{1-\omega^{-j}}\right)\right] \\
= & \phi^{0}, \\
\eta^{l} p_{0}+ & \eta^{l} \omega^{l} p_{1}+\eta^{l} \omega^{2 l} p_{2}+\cdots+\eta^{l} \omega^{(n-1) l} p_{n-1} \\
= & \frac{1}{n}\left[\sum_{j=0}^{n-1} \eta^{l} \phi^{j}+\sum_{j=0}^{n-1} \eta^{l} \phi^{j}+\cdots+\sum_{j=0}^{n-1} \eta^{l} \phi^{j}\right] \\
= & \phi^{l} .
\end{aligned}
$$

Finally, we gain

$$
\begin{gathered}
p_{l}\left(x_{0} I+x_{1} \pi+x_{2} \pi^{2}+\cdots+x_{n-1} \pi^{n-1}\right) \\
=\frac{1}{n} \sum_{j=0}^{n-1} \eta^{-j} \omega^{-l j} \phi^{j}(S)
\end{gathered}
$$




$$
\begin{aligned}
& =\frac{1}{n}\left[x_{0} I+x_{1} \pi+x_{2} \pi^{2}+\cdots+x_{n-1} \pi^{n-1}\right. \\
& +\eta^{-1} \omega^{-l}\left(\eta x_{0} I+\eta \omega x_{1} \pi+\cdots+\eta \omega^{n-1} x_{n-1} \pi^{n-1}\right) \\
& +\cdots+\eta^{-(n-1)} \omega^{-(n-1) l}\left(\eta^{n-1} x_{0} I+\eta^{n-1} \omega^{n-1} x_{1} \pi\right. \\
& \left.\left.+\cdots+\eta^{n-1} \omega^{(n-1)^{2}} x_{n-1} \pi^{n-1}\right)\right]
\end{aligned}
$$$$
=x_{l} \pi^{l} \text {. }
$$

Synthesizing Propositions 1, 2, and 3, we get the following theorem.

Theorem 4. The algebras generated by $I, \pi, \pi^{2}, \ldots, \pi^{n-1}$ and $\phi^{0}, \phi^{1}, \phi^{2}, \ldots, \phi^{n-1}$ over $\mathbf{C}$ are isomorphic and can be called skew-circulant algebras.

\section{Functional Equations for the Skew-Circulant Algebra}

Proposition 5. If $f$ is a linear entire function, $f: \mathbf{C} \rightarrow \mathbf{C}$, then

$$
\begin{aligned}
f\left(c_{0} I\right. & \left.+c_{1} \pi+\cdots+c_{n-1} \pi^{n-1}\right) \\
& =\sum_{l=0}^{n-1}\left[\frac{1}{n} \sum_{j=0}^{n-1} \eta^{-j} \omega^{-l j} f\left(\sum_{k=0}^{n-1} \eta^{k} \omega^{j k} c_{k}\right)\right] \pi^{l} .
\end{aligned}
$$

Proof. According to [3], we know that

$$
\begin{aligned}
& \lambda_{l}=\sum_{j=0}^{n-1} \eta^{j} \omega^{l j} c_{j}, \\
& c_{l}=\frac{1}{n} \sum_{j=0}^{n-1} \eta^{-j} \omega^{-l j} \lambda_{j},
\end{aligned}
$$

for $l=0,1, \ldots, n-1$.

We thus have

$$
\begin{aligned}
\sum_{l=0}^{n-1} f\left(c_{l}\right) \pi^{l} & =\sum_{l=0}^{n-1} f\left(\frac{1}{n} \sum_{j=0}^{n-1} \eta^{-j} \omega^{-l j} \lambda_{j}\right) \pi^{l} \\
& =\sum_{l=0}^{n-1} \frac{1}{n} \sum_{j=0}^{n-1} \eta^{-j} \omega^{-l j} f\left(\lambda_{j}\right) \pi^{l} \\
& =\sum_{l=0}^{n-1}\left[\frac{1}{n} \sum_{j=0}^{n-1} \eta^{-j} \omega^{-l j} f\left(\sum_{k=0}^{n-1} \eta^{k} \omega^{j k} c_{k}\right)\right] \pi^{l}
\end{aligned}
$$

Then, we complete the proof of this conclusion.
For any linear entire function $f: \mathbf{C} \rightarrow \mathbf{C}$, (15) can be written as follows:

$$
\begin{aligned}
f\left(c_{0} I\right. & \left.+c_{1} \pi+\cdots+c_{n-1} \pi^{n-1}\right) \\
& =\sum_{l=0}^{n-1} F_{l}\left(c_{0}, c_{1}, \ldots, c_{n-1}\right) \pi^{l}
\end{aligned}
$$

where

$$
\begin{aligned}
& F_{l}\left(c_{0}, c_{1}, \ldots, c_{n-1}\right) \\
& \quad=\frac{1}{n} \sum_{k=0}^{n-1} \eta^{-k} \omega^{-l k} f\left(\sum_{j=0}^{n-1} \eta^{j} \omega^{j k} c_{j}\right),
\end{aligned}
$$

$l=0,1,2, \ldots, n-1$.

For each $l, F_{0}, F_{1}, \ldots, F_{n-1}$ satisfy the functional equation

$$
\begin{gathered}
F_{l}\left(\eta c_{0}, \eta \omega c_{1}, \eta \omega^{2} c_{2}, \ldots, \eta \omega^{n-1} c_{n-1}\right) \\
=\eta \omega^{l} F_{l}\left(c_{0}, c_{1}, \ldots, c_{n-1}\right)
\end{gathered}
$$

for $\eta=e^{\pi i / n}, \omega=e^{2 \pi i / n}$, all $l=0,1,2, \ldots, n-1$, and all $c_{0}, c_{1}, \ldots, c_{n-1} \in \mathbf{C}$.

Equation (20) is related to the skew-circulant algebra also in another way.

If the function $F$ is defined by

$$
F\left(c_{0}, c_{1}, \ldots, c_{n-1}\right)=\frac{1}{n} \sum_{k=0}^{n-1} \eta^{-k} \omega^{-l k} f\left(\sum_{j=0}^{n-1} \eta^{j} \omega^{j k} c_{j}\right)
$$

let $U=\left\{F \mid F: \mathbf{C}^{n} \rightarrow \mathbf{C}\right\}$ and let $O$ be the operator $O: U \rightarrow$ $U$, linear in $F$, defined by

$$
\begin{aligned}
O(F) & \left(c_{0}, c_{1}, \ldots, c_{n-1}\right) \\
& =F\left(\eta c_{0}, \eta \omega c_{1}, \eta \omega^{2} c_{2}, \ldots, \eta \omega^{n-1} c_{n-1}\right) ;
\end{aligned}
$$

that is,

$$
O(F)=\eta \omega^{l} F
$$

If we denote $O^{k}$ the operation $O$ composed with itself $k$ times, then

$$
\begin{aligned}
O^{k}(F) & \left(c_{0}, c_{1}, c_{2}, \ldots, c_{n-1}\right) \\
= & F\left(\eta^{k} c_{0}, \eta^{k} \omega^{k} c_{1}, \ldots, \eta^{k} \omega^{(n-1) k} c_{n-1}\right),
\end{aligned}
$$

where $O^{j}=-O^{0}$ if and only if $n$ divides $j$ and $j / n$ is an odd number; $O^{j}=O^{0}$ if and only if $n$ divides $j$ and $j / n$ is an even number. 
All these equations (15)-(24) lead up to the following theorem.

Theorem 6. Linear combinations of the operators $\mathrm{O}^{0}, \mathrm{O}^{1}, \ldots, \mathrm{O}^{n-1}$ over $\mathbf{C}$ form a skew-circulant algebra.

Proposition 7. If the operators $R_{0}, R_{1}, \ldots, R_{n-1}: U \rightarrow U$ are defined by

$$
\begin{aligned}
R_{l}=\frac{1}{n}( & O^{0}+\eta^{-1} \omega^{-l} O^{1}+\eta^{-2} \omega^{-2 l} O^{2} \\
& \left.+\cdots+\eta^{-(n-1)} \omega^{-(n-1) l} O^{n-1}\right),
\end{aligned}
$$

for $l=0,1,2, \ldots, n-1$, then these operators $R_{l}(l=$ $0,1,2, \ldots, n-1)$ have the following properties:

$$
\begin{gathered}
R_{l}^{2}=R_{l}, \quad \text { for } l=0,1,2, \ldots, n-1 ; \\
R_{l} R_{j}=0 \quad \text { if } l \neq j ; \\
R_{0}+R_{1}+\cdots+R_{n-1}=O^{0} ; \\
R_{0}+\eta \omega^{l} R_{1}+\cdots+\eta^{n-1} \omega^{(n-1) l} R_{n-1}=O^{l},
\end{gathered}
$$

for $l=0,1,2, \ldots, n-1$.

Through simple calculation, we can get properties (26)(28). Properties (26)-(29) are similar to those of the functions $G_{0}, G_{1}, \ldots, G_{n-1}$ and $p_{0}, p_{1}, \ldots, p_{n-1}$.

By all accounts, a function $F$ in $U$ satisfies (20) if and only if $F \in \operatorname{Ran} R_{l}$ (the range of the operator $R_{l}$ was denoted by $\left.\operatorname{Ran} R_{l}\right)$. Moreover, properties (26)-(28) above imply that $U=\operatorname{Ran}_{0} \oplus \operatorname{Ran} R_{1} \oplus \cdots \oplus \operatorname{Ran} R_{n-1}$, each function $F_{l}$, $l=0,1,2, \ldots, n-1$, defined by (19) is in $\operatorname{Ran} R_{l}$.

In addition,

$$
\begin{aligned}
F_{0}+ & F_{1}+\cdots+F_{n-1} \\
& =f\left(c_{0}+\eta c_{1}+\cdots+\eta^{n-1} c_{n-1}\right) .
\end{aligned}
$$

Thus

$$
F_{l}=R_{l}\left(f\left(c_{0}+\eta c_{1}+\cdots+\eta^{n-1} c_{n-1}\right)\right)
$$

\section{Other Skew-Circulant Algebras}

By (25) and (24), if a function $g$ maps $\mathbf{C}^{n}$ into $\mathbf{C}$, then

$$
\begin{gathered}
R_{l}(g)\left(c_{0}, c_{1}, \ldots, c_{n-1}\right) \\
=\frac{1}{n} \sum_{k=0}^{n-1} \eta^{-k} \omega^{-l k} g\left(\eta^{k} c_{0}+\eta^{k} \omega c_{1}+\eta^{k} \omega^{2 k} c_{2}+\cdots\right. \\
\left.+\eta^{k} \omega^{(n-1) k} c_{n-1}\right)
\end{gathered}
$$

for $l=0,1,2, \ldots, n-1$.
For $f: S_{n} \rightarrow S_{n}, S_{n}$ is the space of skew-circulant matrices, there exist functions $f_{0}, f_{1}, f_{2}, \ldots, f_{n-1}: \mathbf{C}^{n} \rightarrow \mathbf{C}$ such that

$$
f\left(\sum_{l=0}^{n-1} c_{l} \pi^{l}\right)=\sum_{l=0}^{n-1} f_{l}\left(c_{0}, c_{1}, c_{2}, \ldots, c_{n-1}\right) \pi^{l}
$$

Hence, from (28),

$$
\begin{aligned}
f\left(\sum_{l=0}^{n-1} c_{l} \pi^{l}\right) & =\sum_{l=0}^{n-1} \sum_{i=0}^{n-1} R_{l+i}\left(f_{l}\right) \pi^{l} \\
& =\sum_{i=0}^{n-1} \sum_{l=0}^{n-1} R_{l+i}\left(f_{l}\right) \pi^{l} .
\end{aligned}
$$

Proposition 8. Let $V=\left\{f \mid f: S_{n} \rightarrow S_{n}, S_{n}\right.$ is the space of skew-circulant matrices $\}$, for $f \in V$; let us define $q_{i}(f)$ and $g_{i}$ such that

$$
\begin{gathered}
q_{i}(f)\left(\sum_{l=0}^{n-1} c_{l} \pi^{l}\right)=\sum_{l=0}^{n-1} R_{l+i}\left(f_{l}\right) \pi^{l}, \\
g_{i}=\sum_{l=0}^{n-1} R_{l+i}\left(f_{l}\right),
\end{gathered}
$$

for $i=0,1,2, \ldots, n-1$ and $l+i$ taken modulo $n$. Then we can receive the properties of the $q_{i}$ :

$$
\begin{gathered}
q_{i}^{2}=q_{i} \quad \text { for } i=0,1,2, \ldots, n-1 ; \\
q_{i} q_{j}=0 \quad \text { if } i \neq j ; \\
\left(q_{0}+q_{1}+q_{2}+\cdots+q_{n-1}\right)(f)=f, \quad f \in V .
\end{gathered}
$$

From what has been discussed above, we gain the following theorem.

Theorem 9. The $q_{0}, q_{1}, q_{2}, \ldots, q_{n-1}$ are orthogonal projections on $V$, adding to the identity function on $V$, and so generating over $\mathbf{C}$ a skew-circulant algebra.

Proposition 10. The projections $q_{i}(f)$ have another formula as follows:

$$
\begin{array}{r}
q_{i}(f)\left(\sum_{l=0}^{n-1} \mathcal{c}_{l} \pi^{l}\right)=\frac{1}{n} \sum_{k=0}^{n-1} \omega^{-i k} \phi^{-k} f \phi^{k}\left(\sum_{l=0}^{n-1} \mathcal{c}_{l} \pi^{l}\right), \\
i=0,1, \ldots, n-1 .
\end{array}
$$


Proof. We can prove (38) in the following:

$$
\begin{aligned}
& q_{i}(f)\left(\sum_{l=0}^{n-1} c_{l} \pi^{l}\right) \\
&= \sum_{l=0}^{n-1} R_{l+i}\left(f_{l}\right) \pi^{l} \\
&= \sum_{l=0}^{n-1}\left[\frac { 1 } { n } \sum _ { k = 0 } ^ { n - 1 } \eta ^ { - k } \omega ^ { - ( l + i ) k } f _ { l } \left(\eta^{k} c_{0}, \eta^{k} \omega^{k} c_{1}, \ldots,\right.\right. \\
&= \frac{1}{n} \sum_{l=0}^{n-1}\left[\sum _ { k = 0 } ^ { n - 1 } \eta ^ { - k } \omega ^ { - l k } \omega ^ { - i k } f _ { l } \left(\eta^{k} c_{0}, \ldots,\right.\right. \\
&=\left.\left.\frac{1}{n} \sum_{k=0}^{n-1} \omega^{k} \omega^{(n-1) k} c_{n-1}\right)\right] \pi^{l} \\
&= \frac{1}{n} \sum_{k=0}^{n-1} \eta^{-k} \omega^{-i k} \sum_{l=0}^{n-1} \omega^{-l k} f_{l}\left(\eta^{k} c_{0}, \eta^{k} \omega^{k} c_{1}, \ldots,\right. \\
&\left.\eta^{k} \phi^{k} \omega^{(n-1) k} c_{n-1}\right) \pi^{l}\left.\sum_{l=0}^{n-1} c_{l} \pi^{l}\right) . \\
&\left.\left.\left.\eta^{k} \omega^{(n-1) k} c_{n-1}\right)\right] \pi^{l} \omega^{l k} c_{l} \pi^{l}\right)
\end{aligned}
$$

This result can be rewritten in the form

$$
q_{i}(f)\left(\sum_{l=0}^{n-1} c_{l} \pi^{l}\right)=\frac{1}{n} \sum_{k=0}^{n-1} \omega^{-i k} \phi^{-k} f \phi^{k},
$$

$i=0,1, \ldots, n-1$, for all functions $f \in V$. $\operatorname{Ran} p_{i}$.

Finally, it can be shown that $f \phi=\omega^{i} \phi f$ if and only if $f \in$

Theorem 11. For each $f \in V$ and every $i=0,1,2, \ldots, n-$ 1 , there exists only one function $g_{i}$ such that the following equation holds:

$$
q_{i}(f)\left(\sum_{l=0}^{n-1} \mathcal{c}_{l} \pi^{l}\right)=\sum_{l=0}^{n-1} R_{l+i}\left(g_{i}\right) \pi^{l},
$$

for $g_{i}=\sum_{l=0}^{n-1} R_{l+i}\left(f_{l}\right), i=0,1,2, \ldots, n-1$ and $l+i$ taken modulo n.

Proof. By (26) and (27),

$$
R_{l+i}\left(g_{i}\right)=R_{l+i}\left(f_{l}\right)
$$

for $l=0,1,2, \ldots, n-1 ; i=0,1,2, \ldots, n-1$ and $l+i$ taken modulo $n$.

Suppose there exists another function $g_{i}^{*}: \mathbf{C}^{n} \rightarrow \mathbf{C}$ such that $q_{i}(f)=\sum_{l=0}^{n-1} R_{l+i}\left(g_{i}\right) \pi^{l}=\sum_{l=0}^{n-1} R_{l+i}\left(g_{i}^{*}\right) \pi^{l}$. Since $I, \pi, \pi^{2}, \ldots, \pi^{n-1}$ is a basis of $S_{n}, R_{l+i}\left(g_{i}\right)=R_{l+i}\left(g_{i}^{*}\right)$ for $l=$ $0,1, \ldots, n-1$. By $(28)$,

$$
g_{i}=\sum_{l=0}^{n-1} R_{l+i}\left(g_{i}\right)=\sum_{l=0}^{n-1} R_{l+i}\left(g_{i}^{*}\right)=g_{i}^{*} .
$$

So $g_{i}$ is unique. Thus, there is an isomorphism between functions $g_{i}: \mathbf{C}^{n} \rightarrow \mathbf{C}$ and functions $\sum_{l=0}^{n-1} R_{l+i}\left(g_{i}\right) \pi^{l}$ in $\operatorname{Ran} q_{i}$.

Now the results of all this are as follows: let $\Phi=\{f \mid f$ : $\mathbf{C} \rightarrow \mathbf{C}$ \} with $f$ an entire function and $U=\left\{f \mid f: \mathbf{C}^{n} \rightarrow\right.$ C). Let $I$ be a monomorphism $\Phi \rightarrow U^{n}$ defined by $I(f)=$ $\left(f\left(c_{0}+\eta c_{1}+\cdots+\eta^{n-1} c_{n-1}\right), 0, \ldots, 0\right)$. Let $\tau$ be a monomorphism $\Phi \rightarrow V$ defined by

$$
\begin{aligned}
& \tau(f)\left(\sum_{l=0}^{n-1} c_{l} \pi^{l}\right) \\
& =\sum_{l=0}^{n-1} R_{n}\left(f\left(c_{0}+\eta c_{1}+\cdots+\eta^{n-1} c_{n-1}\right)\right) \pi^{l} \\
& =f\left(\sum_{l=0}^{n-1} c_{l} \pi^{l}\right),
\end{aligned}
$$

which follows from (18), (19), and (31). Then there exists an isomorphism $\theta: U^{n} \rightarrow V$ defined by

$$
\begin{gathered}
\theta\left(g_{0}, g_{1}, g_{2}, \ldots, g_{n-1}\right)\left(\sum_{l=0}^{n-1} c_{l} \pi^{l}\right) \\
=\sum_{i=0}^{n-1} \sum_{l=0}^{n-1} R_{l+i}\left(g_{i}\right) \pi^{l} .
\end{gathered}
$$

\section{A Linear Involution}

Suppose that $g$ is a function $S_{n} \rightarrow S_{n}$ given by

$$
g=\sum_{i=0}^{n-1} g_{i} \pi^{i}
$$

where $g_{i}$ is given by (36). Written out, we have

$$
g=\sum_{i=0}^{n-1}\left[\sum_{l=0}^{n-1} R_{l+i}\left(f_{l}\right)\right] \pi^{i} .
$$

Let $V$ denote the space of functions $f: S_{n} \rightarrow S_{n}$. If $f$ is an element of $V$, then there exist a set of $n$ functions $f_{0}, f_{1}, \ldots, f_{n-1}$ mapping $\mathbf{C}^{n}$ into $\mathbf{C}$ such that $f=\sum_{l=0}^{n-1} f_{l} \pi^{l}$ (like (33)). Let us switch the $l$ and the $i$ in the right-hand side of (47). Then let $\psi$ be the function $V \rightarrow V$ defined by

$$
\psi\left(\sum_{l=0}^{n-1} f_{l} \pi^{l}\right)=\sum_{l=0}^{n-1}\left(\sum_{i=0}^{n-1} R_{l+i}\left(f_{i}\right)\right) \pi^{l} .
$$


Theorem 12. From (26), (27), and (28), we can show that $\psi$ is a linear involution on $V$.

Proof. From (26), (27), and (28), we get

$$
\begin{aligned}
\psi\left(\psi\left(\sum_{l=0}^{n-1} f_{l} \pi^{l}\right)\right) & =\sum_{l=0}^{n-1}\left\{\sum_{i=0}^{n-1} R_{l+i}\left[\sum_{k=0}^{n-1} R_{i+k}\left(f_{k}\right)\right]\right\} \pi^{l} \\
& =\sum_{l=0}^{n-1}\left[\sum_{i=0}^{n-1} R_{l+i}\left(f_{l}\right)\right] \pi^{l} \\
& =\sum_{l=0}^{n-1} f_{l} \pi^{l} .
\end{aligned}
$$

That is, $\psi^{2}=\psi^{0}$ (the identity function on $V$ ). Then, $\psi$ is a linear involution on $V$.

Consider the set $W=\left\{\alpha \psi^{0}+\beta \psi \mid \alpha, \beta \in \mathbf{C}\right\}$, that is, linear combinations over $\mathbf{C}$ of $\psi^{0}$ and $\psi\left(\right.$ since $\left.\psi^{2}=\psi^{0}\right)$. Then $W$ is a $2 \times 2$ complex circulant algebra; $\left(\psi^{0}+\psi\right) / 2$ and $\left(\psi^{0}-\psi\right) / 2$ are idempotent elements of $W$; that is, they are projections on $V$. If $f$ is in $V$, then $\psi(f)=f$ if and only if $f \in \operatorname{Ran}\left(\psi^{0}+\psi\right) / 2$, and $\psi(f)=-f$ if and only if $f \in \operatorname{Ran}\left(\psi^{0}-\psi\right) / 2$. Also, $V=$ $\left(\operatorname{Ran}\left(\psi^{0}+\psi\right) / 2\right) \oplus\left(\operatorname{Ran}\left(\psi^{0}-\psi\right) / 2\right)($ a direct sum $)$.

Example 13. If $n=2$, then $\pi^{2}=-I$. Let $f$ and $g$ be two functions $\mathbf{C}^{2} \rightarrow \mathbf{C}$. Then

$$
\begin{aligned}
& \psi\left(\operatorname{If}\left(c_{0}, c_{1}\right)+\pi g\left(c_{0}, c_{1}\right)\right) \\
& \begin{aligned}
&= \frac{I}{2}\left[f\left(c_{0}, c_{1}\right)-i f\left(i c_{0},-i c_{1}\right)+g\left(c_{0}, c_{1}\right)+i g\left(i c_{0},-i c_{1}\right)\right] \\
&+\frac{\pi}{2}\left[f\left(c_{0}, c_{1}\right)+i f\left(i c_{0},-i c_{1}\right)\right. \\
&\left.+g\left(c_{0}, c_{1}\right)-g\left(-c_{0},-c_{1}\right)\right] .
\end{aligned}
\end{aligned}
$$

Note that, if $f_{i}$ is a function $\mathbf{C}^{n} \rightarrow \mathbf{C}$ for each $i$, we have by (48) that

$$
\psi\left(f_{i} \pi^{i}\right)=\sum_{l=0}^{n-1} R_{l+i}\left(f_{i}\right) \pi^{l}
$$

In the same way, (48) implies that

$$
\psi\left[R_{l+i}\left(f_{l}\right) \pi^{l}\right]=R_{l+i}\left(f_{l}\right) \pi^{i}
$$

where $l$ and $i$ vary from 0 to $n-1$ and $l+i$ is taken modulo $n$. Since $\psi(f)=f$ if and only if $f \in \operatorname{Ran}\left(\psi^{0}+\psi\right) / 2$, the function

$$
\begin{aligned}
\left(\frac{1}{2}\right) & \left(\psi^{0}+\psi\right)\left[R_{l+i}\left(f_{l}\right) \pi^{l}\right] \\
& =\left(\frac{1}{2}\right) R_{l+i}\left(f_{l}\right)\left(\pi^{l}+\pi^{i}\right)
\end{aligned}
$$

is a fixed point of $\psi$.

\section{Conclusion}

We prove that the set of skew-circulants with complex entries has an idempotent basis. This paper displays algebras of operators which are isomorphic to the algebra of $n \times n$ complex skew-circulant matrices. In [19], a new fast algorithm for optimal design of block digital filters (BDFs) is proposed based on skew circulant matrix. The reason why we focus our attention on skew-circulant operator is to explore the application of skew-circulant in the related field. On the basis of existing application situation [2-8], we will exploit solving ordinary, partial, and delay differential equations based on skew circulant operator.

\section{Conflict of Interests}

The authors declare that there is no conflict of interests regarding the publication of this paper.

\section{Acknowledgments}

The research is supported by the Development Project of Science \& Technology of Shandong Province (Grant no. 2012GGX10115) and NSFC (Grant no. 11301251) and the AMEP of Linyi University, China.

\section{References}

[1] D. Bertaccini and M. K. Ng, "Skew-circulant preconditioners for systems of LMF-based ODE codes," in Numerical Analysis and Its Applications, vol. 1988 of Lecture Notes in Computer Science, pp. 93-101, Springer, Berlin, Germany, 2001.

[2] J. Delgado, N. Romero, A. Rovella, and F. Vilamajó, "Bounded solutions of quadratic circulant difference equations," Journal of Difference Equations and Applications, vol. 11, no. 10, pp. 897907, 2005.

[3] A. C. Wilde, "Differential equations involving circulant matrices," Rocky Mountain Journal of Mathematics, vol. 13, no. 1, pp. 1-13, 1983.

[4] B. Karasözen and G. Şimşek, "Energy preserving integration of bi-Hamiltonian partial differential equations," Applied Mathematics Letters, vol. 26, no. 12, pp. 1125-1133, 2013.

[5] E. W. Sachs and A. K. Strauss, "Efficient solution of a partial integro-differential equation in finance," Applied Numerical Mathematics, vol. 58, no. 11, pp. 1687-1703, 2008.

[6] R. W. Brockett and J. L. Willems, "Discretized partial differential equations: examples of control systems defined on modules," Automatica, vol. 10, no. 5, pp. 507-515, 1974.

[7] R. D. Nussbaum, "Circulant matrices and differential-delay equations," Journal of Differential Equations, vol. 60, no. 2, pp. 201-217, 1985.

[8] X.-Q. Jin, S.-L. Lei, and Y.-M. Wei, "Circulant preconditioners for solving differential equations with multidelays," Computers \& Mathematics with Applications, vol. 47, no. 8-9, pp. 1429-1436, 2004.

[9] P. J. Davis, Circulant Matrices, John Wiley \& Sons, New York, NY, USA, 1979.

[10] Z. L. Jiang and Z. X. Zhou, Circulant Matrices, Chengdu Technology University, Chengdu, China, 1999. 
[11] R. H. Chan and X.-Q. Jin, "Circulant and skew-circulant preconditioners for skew-Hermitian type Toeplitz systems," BIT Numerical Mathematics, vol. 31, no. 4, pp. 632-646, 1991.

[12] R. H. Chan and K.-P. Ng, "Toeplitz preconditioners for Hermitian Toeplitz systems," Linear Algebra and Its Applications, vol. 190, pp. 181-208, 1993.

[13] T. Huckle, "Circulant and skewcirculant matrices for solving Toeplitz matrix problems," SIAM Journal on Matrix Analysis and Applications, vol. 13, no. 3, pp. 767-777, 1992.

[14] J. N. Lyness and T. Sørevik, "Four-dimensional lattice rules generated by skew-circulant matrices," Mathematics of Computation, vol. 73, no. 245, pp. 279-295, 2004.

[15] H. Karner, J. Schneid, and C. W. Ueberhuber, "Spectral decomposition of real circulant matrices," Linear Algebra and Its Applications, vol. 367, pp. 301-311, 2003.

[16] M. J. Narasimha, "Linear convolution using skew-cyclic convolutions," IEEE Signal Processing Letters, vol. 14, no. 3, pp. 173-176, 2007.

[17] V. C. Liu and P. P. Vaidyanathan, "Circulant and skew-circulant matrices as new normal-form realization of IIR digital filters," IEEE Transactions on Circuits and Systems, vol. 35, no. 6, pp. 625-635, 1988.

[18] J. Li, Z. L. Jiang, N. Shen, and J. W. Zhou, “On optimal backward perturbation analysis for the linear system with skew circulant coefficient matrix," Computational and Mathematical Methods in Medicine, vol. 2013, Article ID 707381, 7 pages, 2013.

[19] D. Q. Fu, Z. L. Jiang, Y. F. Cui, and S. T. Jhang, "A new fast algorithm for optimal design of block digital filters by skewcyclicconvolution," IET Signal Processing.

[20] Y. Gao, Z. L. Jiang, and Y. P. Gong, "On the determinants and inverses of skew circulant and skew left circulant matrices with Fibonacci and Lucas Numbers," WSEAS Transactions on Mathematics, vol. 12, no. 4, pp. 472-481, 2013.

[21] A. C. Wilde, "Solutions of equations containing primitive roots of unity," Journal of Undergraduate Mathematics, vol. 3, pp. 25$28,1971$.

[22] A. C. Wilde, "Cauchy-Riemann conditions for algebras isomorphic to the circulant algebra," The Journal of the University of Kuwait (Science), vol. 14, no. 2, pp. 189-204, 1987.

[23] A. C. Wilde, "Commutative projection operators," Atti del Seminario Matematico e Fisico dell'Università di Modena, vol. 35, no. 1, pp. 167-172, 1987.

[24] A. C. Wilde, "Algebras of operators isomorphic to the circulant algebra," Proceedings of the American Mathematical Society, vol. 105, no. 4, pp. 808-816, 1989.

[25] R. H. Chan, X.-Q. Jin, and M.-C. Yeung, "The circulant operator in the banach algebra of matrices," Linear Algebra and Its Applications, vol. 149, pp. 41-53, 1991.

[26] D. Chillag, "Regular representations of semisimple algebras, separable field extensions, group characters, generalized circulants, and generalized cyclic codes," Linear Algebra and Its Applications, vol. 218, pp. 147-183, 1995.

[27] C. Brink and J. Pretorius, "Boolean circulants, groups, and relation algebras," The American Mathematical Monthly, vol. 99, no. 2, pp. 146-152, 1992.

[28] R. H. Chan, X.-Q. Jin, and M. K. Ng, "Circulant integral operators as preconditioners for Wiener-Hopf equations," Integral Equations and Operator Theory, vol. 21, no. 1, pp. 12-23, 1995.

[29] F. di Benedetto and S. serra Capizzano, "Optimal multilevel matrix algebra operators," Linear and Multilinear Algebra, vol. 48 , no. 1, pp. 35-66, 2000.
[30] F. di Benedetto and S. serra Capizzano, "A note on the superoptimal matrix algebra operators," Linear and Multilinear Algebra, vol. 50, no. 4, pp. 343-372, 2002.

[31] I. S. Hwang, D.-O. Kang, and W. Y. Lee, "Hyponormal Toeplitz operators with matrix-valued circulant symbols," Complex Analysis and Operator Theory, vol. 7, no. 4, pp. 843-861, 2013.

[32] W. Bani-Domi and F. Kittaneh, "Norm equalities and inequalities for operator matrices," Linear Algebra and Its Applications, vol. 429, no. 1, pp. 57-67, 2008. 


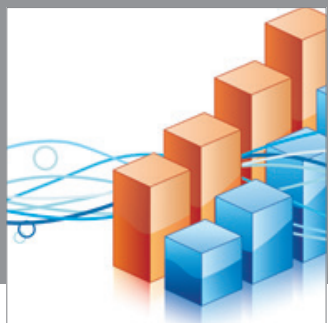

Advances in

Operations Research

mansans

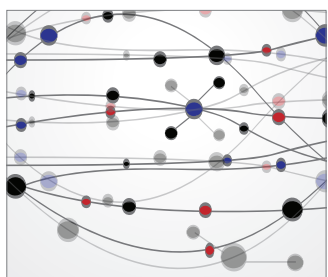

The Scientific World Journal
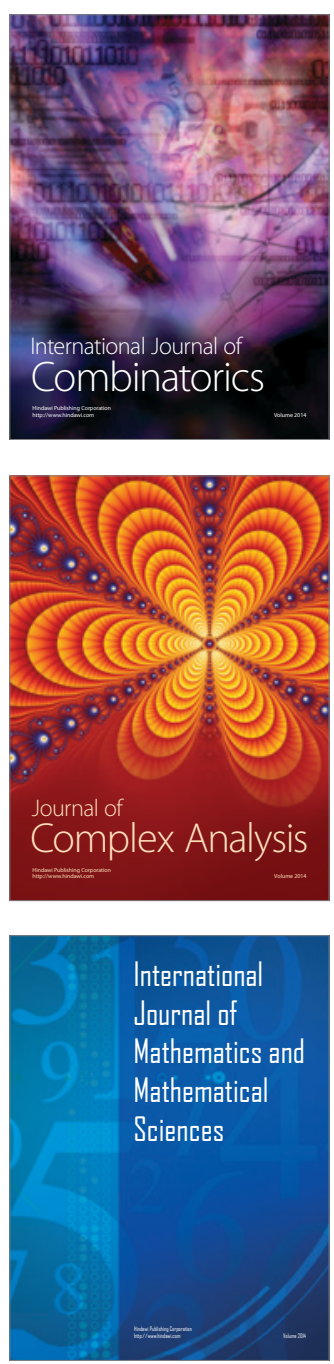
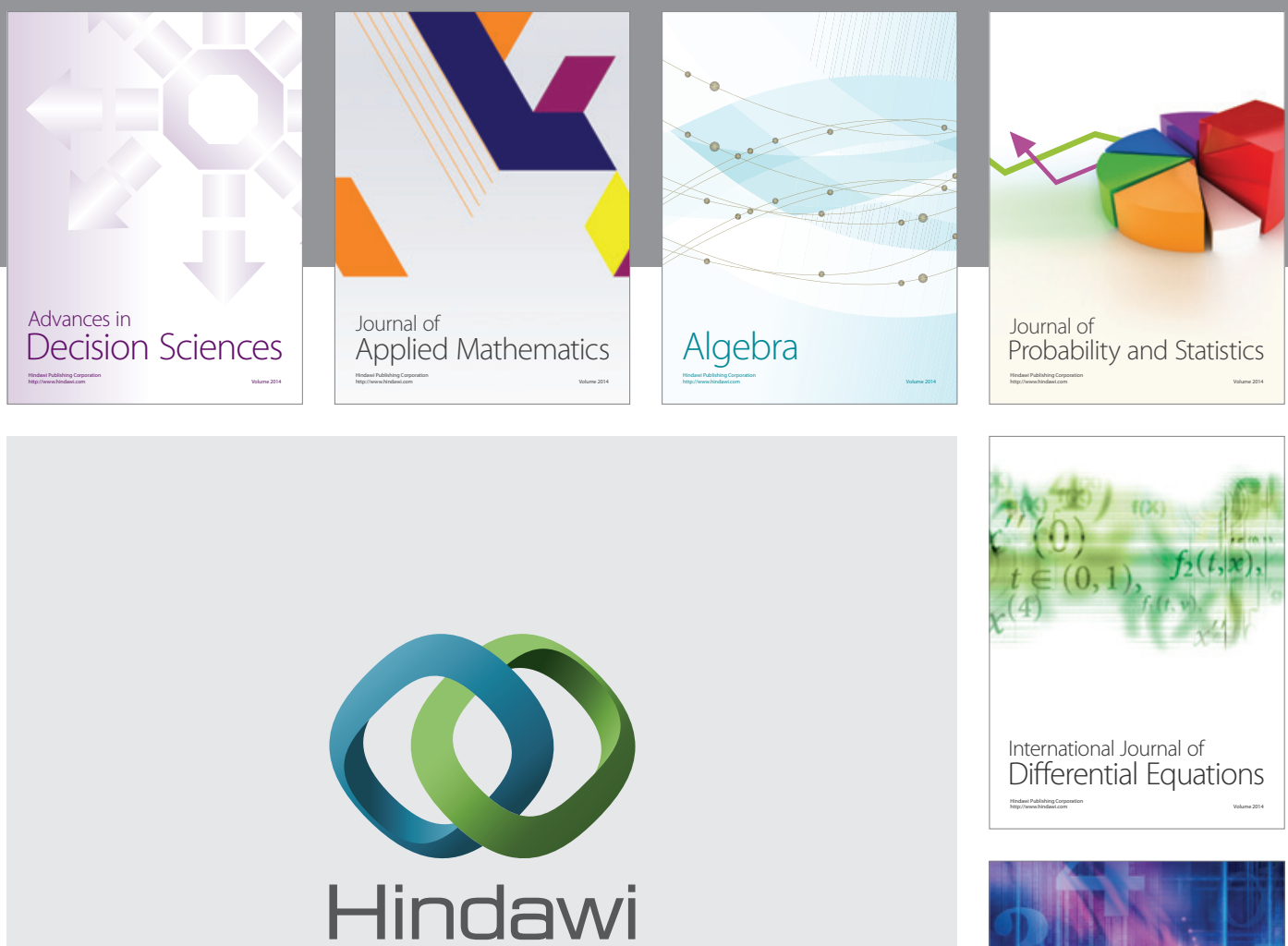

Submit your manuscripts at http://www.hindawi.com
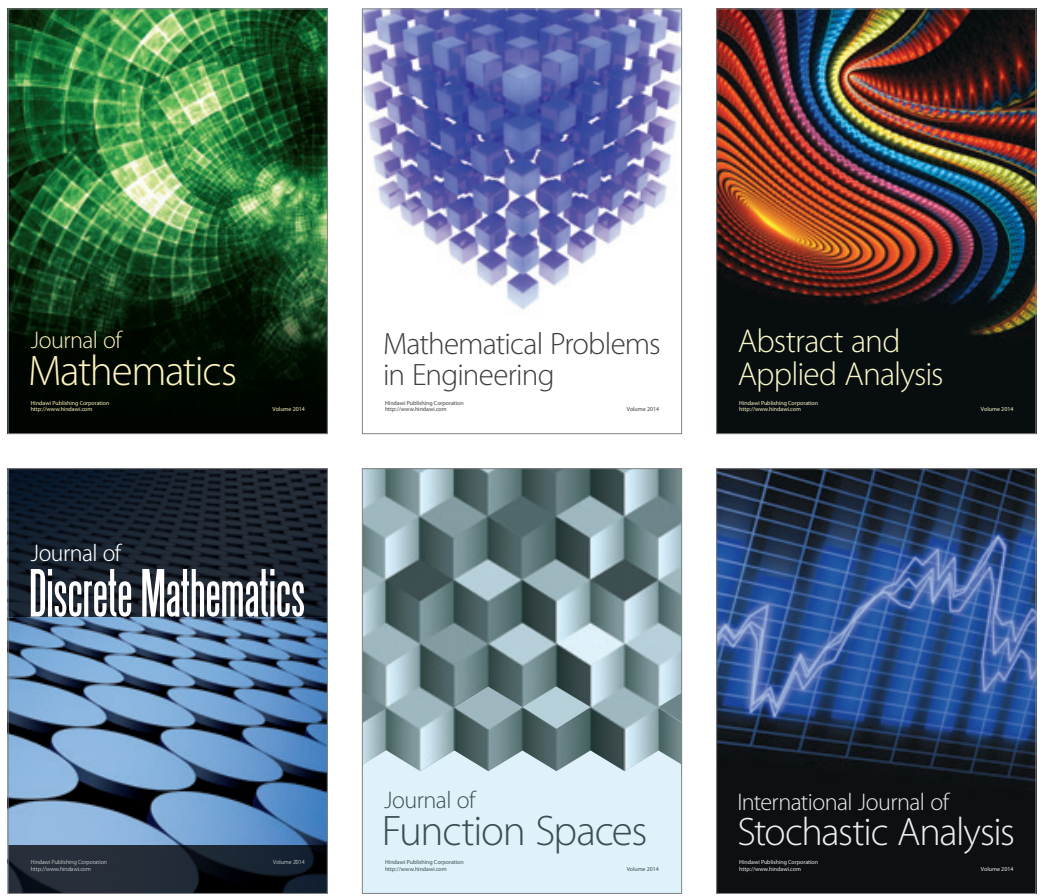

Journal of

Function Spaces

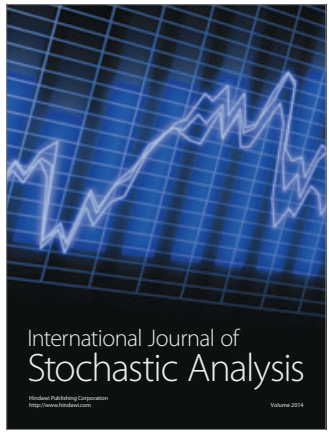

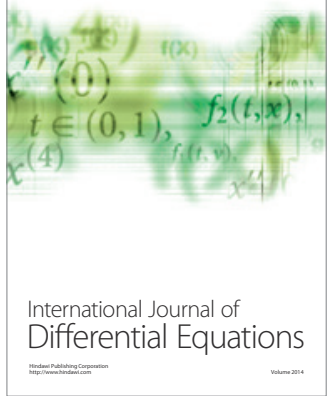
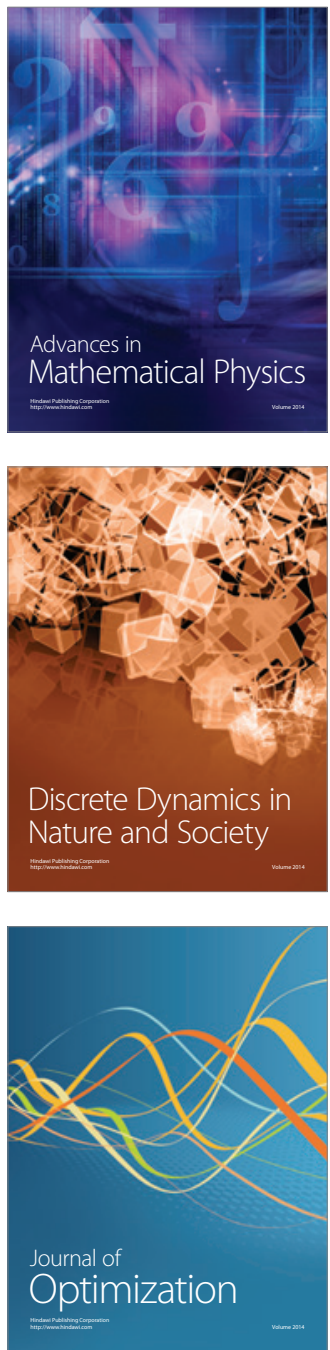